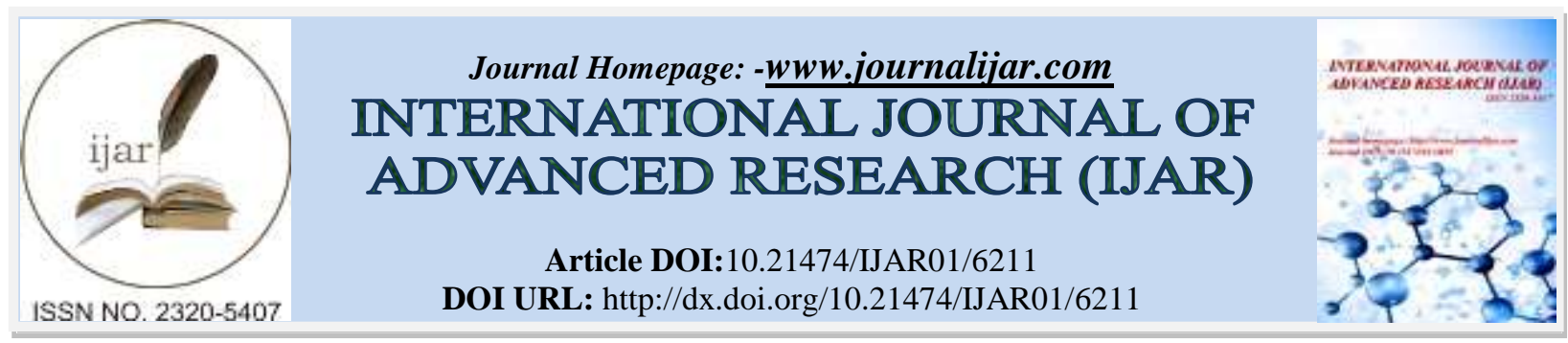

RESEARCH ARTICLE

\title{
A RARE CASE REPORT OF PLEOMORPHIC ADENOMA ARISING FROM MINOR SALIVARY GLAND.
}

Dr. Neethu Telagi ${ }^{1}$, Dr. Ahmed Mujib. B. $\mathbf{R}^{1}$ and Dr. Manasa Syamala ${ }^{2}$.

1. Department of Oral Pathology \& Microbiology, Bapuji Dental College and Hospital.

2. Consultant Doctor, Maneesha Dental Care and Implant Centre, Pinnamaneni Polyclinic Road, Vijayawada 520001, Andhra Pradesh.

\section{Manuscript Info}

\section{Manuscript History}

Received: 04 November 2017

Final Accepted: 06 December 2017

Published: January 2018

Key words:-

Buccal mucosa, minor salivary glands, swelling, pleomorphic adenoma.

\section{Abstract}

Salivary gland tumors account for less than three percent of the head and neck tumors. Among all salivary gland tumors, pleomorphic adenoma is the most frequently encountered lesion, accounting for approximately $60 \%$ of all salivary gland neoplasms. Pleomorphic adenoma is the most common benign neoplasm of the salivary glands but it is the rare tumor involving minor salivary glands. Here we present a rare case report of buccal mucosa swelling which was found to be pleomorphic adenoma arising from the minor salivary glands in the buccal mucosa.

Copy Right, IJAR, 2018,. All rights reserved.

\section{Introduction:-}

Salivary gland tumors account for less than three percent of the head and neck tumors. Among all salivary gland tumors, pleomorphic adenoma is the most frequently encountered lesion, accounting for approximately $60 \%$ of all salivary gland neoplasms. ${ }^{1}$

Pleomorphic adenoma is the most common benign neoplasm of the salivary glands. The palate is considered as the most common intraoral site (42.8-68.8\%), followed by the upper lip (10.1\%) and cheek (5.5\%). Other rare sites include the throat $(2.5 \%)$, retromolar region $(0.7 \%)$, floor of the mouth and the alveolar mucosa. ${ }^{2}$

This report describes a case of pleomorphic adenoma arising in the cheek, which underwent therapeutic wide local excision with wide margins. Although pleomorphic adenomas arising from the minor salivary glands rarely affect the cheeks, the condition should still be considered in the differential diagnosis of cheek masses.

\section{Case report:-}

A 48 year old female patient has reported with a chief complaint of swelling on the left buccal mucosa since 10 days. On intra-oral examination, a solitary dome shaped swelling was evident over left back tooth region of buccal mucosa measuring about $1.5 \times 3.0 \mathrm{~cm}$ in diameter in relation to 27,28 and 37,38 region. An ulcer was evident over the swelling measuring about $0.5 \mathrm{~cm}$ in relation to occlusal aspect of distobuccal cusps of 27 and 37 . On palpation the swelling and ulcer were tender and the swelling was mobile and firm in consistency. A provisional diagnosis of traumatic fibroma on left buccal mucosa associated with traumatic ulcer was given. An excisional biopsy of the lesion was performed and the tissue specimen was sent for histological examination which was then routinely processed and stained. The haematoxylin and eosin stained sections from the submitted soft tissue specimen revealed the presence of parakeratinized stratified squamous epithelium and underlying connective tissue stroma. 
The subepithelial connective tissue exhibits the presence of glandular epithelium and myoepithelial cells arranged as ducts and sheets in a myxomatous background. There is also evidence of hyalinised and chondroid areas and focal inflammatory cell infiltrate chiefly consisting of lymphocytes and plasma cells. A final diagnosis of pleomorphic adenoma of minor salivary gland was given.

\section{Discussion:-}

Neoplasms of the salivary glands account for less than $1 \%$ of all tumours, and 3\%-5\% of all head and neck tumours. Around $70 \%$ of these tumours occur in the parotid gland, $10 \%$ in the submandibular gland and the remainder in the minor salivary glands. ${ }^{3}$ Pleomorphic adenomas, in general, comprise $40 \%$ of minor salivary gland tumors (Torske, 2006). They may arise in the nasal cavity, paranasal sinuses, and larynx (Seifert, 1996; Compagno, 1977). ${ }^{4}$ World Health Organization (1972) defined PA as a well-defined tumor characterized by its pleomorphic or mixed appearance. There is intermixing of the clearly recognizable epithelial component with mucoid, myxoid and chondroid component. ${ }^{5}$ Pleomorphic adenoma consists of cells with epithelial and mesenchymal differentiation (mixed tumor).The highly variable morphology of this neoplasm is the result of interplay between these elements. Now it is widely accepted that both epithelial and mesenchymal (myxoid, hyaline, chondroid, osseous) elements often arise from same cell clone, which may be a myoepithelial or ductal reserve cell. There is no difference in behavior of this neoplasm based on proportion of various elements. ${ }^{6}$

Most intra-oral pleomorphic adenomas develop in the minor salivary glands of the palate, followed by the glands in the upper lip and buccal mucosa. Pleomorphic adenomas that have been described in the buccal mucosa are considered to have arisen in the submucosal buccal minor salivary glands. Most minor salivary gland tumours present as a smooth, submucosal mass or nodule and the neoplasm's rate of growth is usually indolent.

The aetiology of pleomorphic adenoma is still not clearly known. However, pleomorphic adenoma is described to have an epithelial origin, along with clonal chromosome abnormalities with aberrations involving 8q12 and $12 \mathrm{q} 15$. Pleomorphic adenomas tend to present as painless, slow growing, submucosal swellings that do not cause fixation or ulceration of the overlying mucosa. The clinical features suggestive of malignant change are sudden increase in size, pain, involvement of skin and deeper layers, and neck adenopathies. ${ }^{3}$ PA occurs commonly between the 3 th and 5 th decades with female predilection. The majority of intraoral mixed tumors are less than $3.0 \mathrm{~cm}$ in diameter. They are usually solitary and well-circumscribed. ${ }^{8}$

The differential diagnosis of the juvenile PA of the cheek includes buccal space abscess, dermoid cyst, foreign body reaction, fibroma, lipoma, neurofibroma, rhabdomyosarcoma, mucoepidermoid carcinoma, adenoid cystic carcinoma, polymorphous low-grade adenocarcinoma and carcinoma ex-PA. The possibility of buccal space abscess was ruled out due to absence of signs of inflammation. The solid nature of the lesion coupled with the lack of tissue representing the three germ layers rule out the possibility of mature cystic teratoma (dermoid cyst). The lack of ulceration of the buccal mucosa, pain, paresthesia or invasion of the surrounding tissue rules out the possibility of malignant transformation. ${ }^{2}$ Absence of punctum and freely movable nature of the mass differentiate PA from sebaceous cyst. As on histological picture both epithelial and myoepithelial cells were seen, which rules out mucoepidermoid carcinoma. The negative slip test clinically and absence of lipomatous component histologically rules out lipoma. The absence of perineural invasion and mitoticfigures obscure the chances of polymorphic low grade adenocarcinoma. ${ }^{8}$

Willis described PA as the lesion with unusual histologic pattern consisting of cells exhibiting the ability to differentiate to epithelial (ductal and nonductal) cells and mesenchymal (chondroid, myxoid and osseous) cells. It demonstrates combinations of glandular epithelium and mesenchyme-like tissue and the proportion of each component varies widely among individual tumors.

Foote and Frazell (1954) categorized the tumor into the following types: Principally myxoid, myxoid and cellular components present in equal proportions, predominantly cellular and extremely cellular. ${ }^{9}$

PA is known to produce recurrence either due to spillage, inadequate removal or enucleation at the time of operation, but is not known to produce distant metastasis. A recurrence rate of $2-44 \%$ has been reported in the literature. The ideal treatment of choice for PA is wide local excision with good safety margins and follows-up for at least 3-4 years. ${ }^{8}$ 


\section{Conclusion:-}

The salivary glands may show a diverse range of lesions presenting a challenge to even the most experienced clinician and pathologist. PA of minor salivary gland is a tumor of rare occurrence and a diagnosis should be made carefully lest a major salivary gland be resected. Pleomorphic adenoma generally does not recur after adequate surgical excision. Ultimately, complete surgical excision will provide the definitive diagnosis and treatment for this noteworthy salivary gland neoplasm.

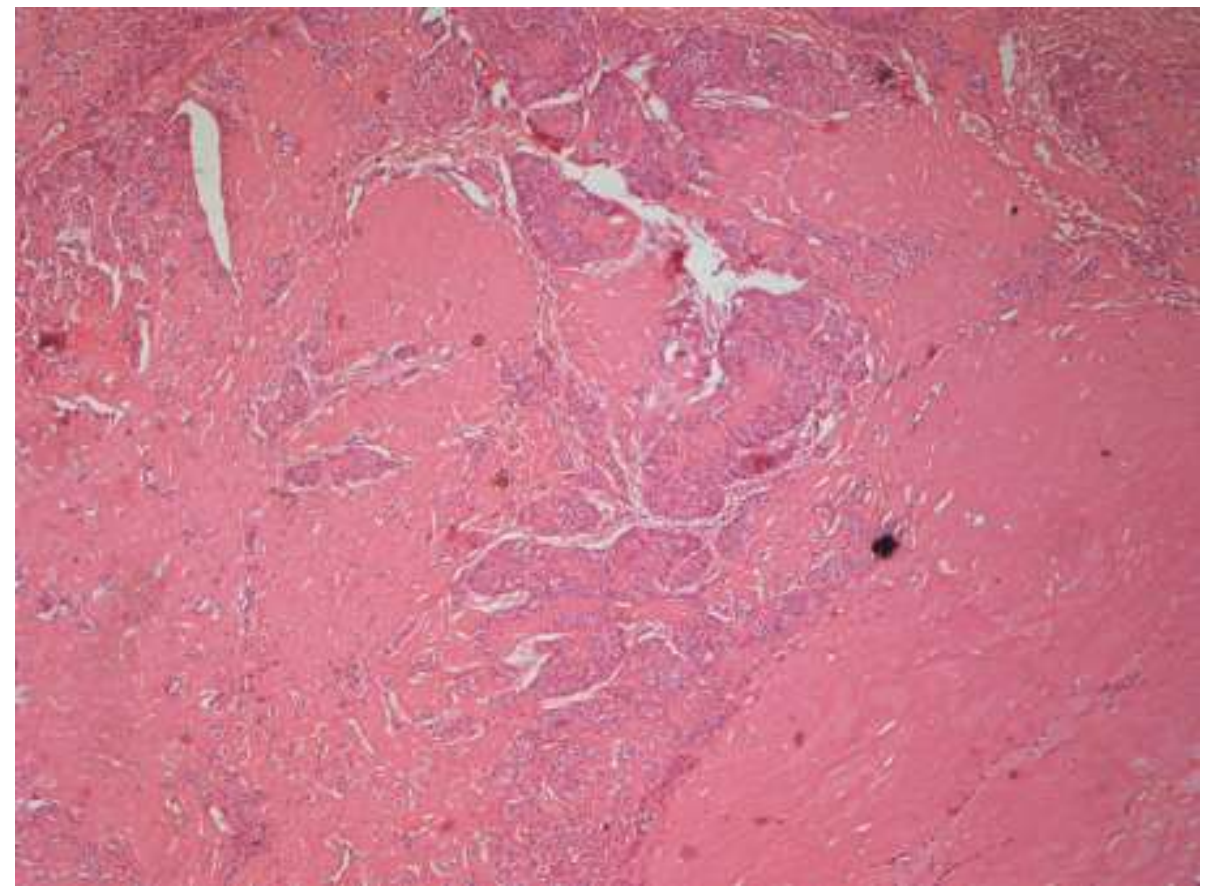

Figure 1:- photomicrograph $(5 \mathrm{x})$ showing myxoid stroma along with myoepithelial cells arranged in the form of strands and small groups.

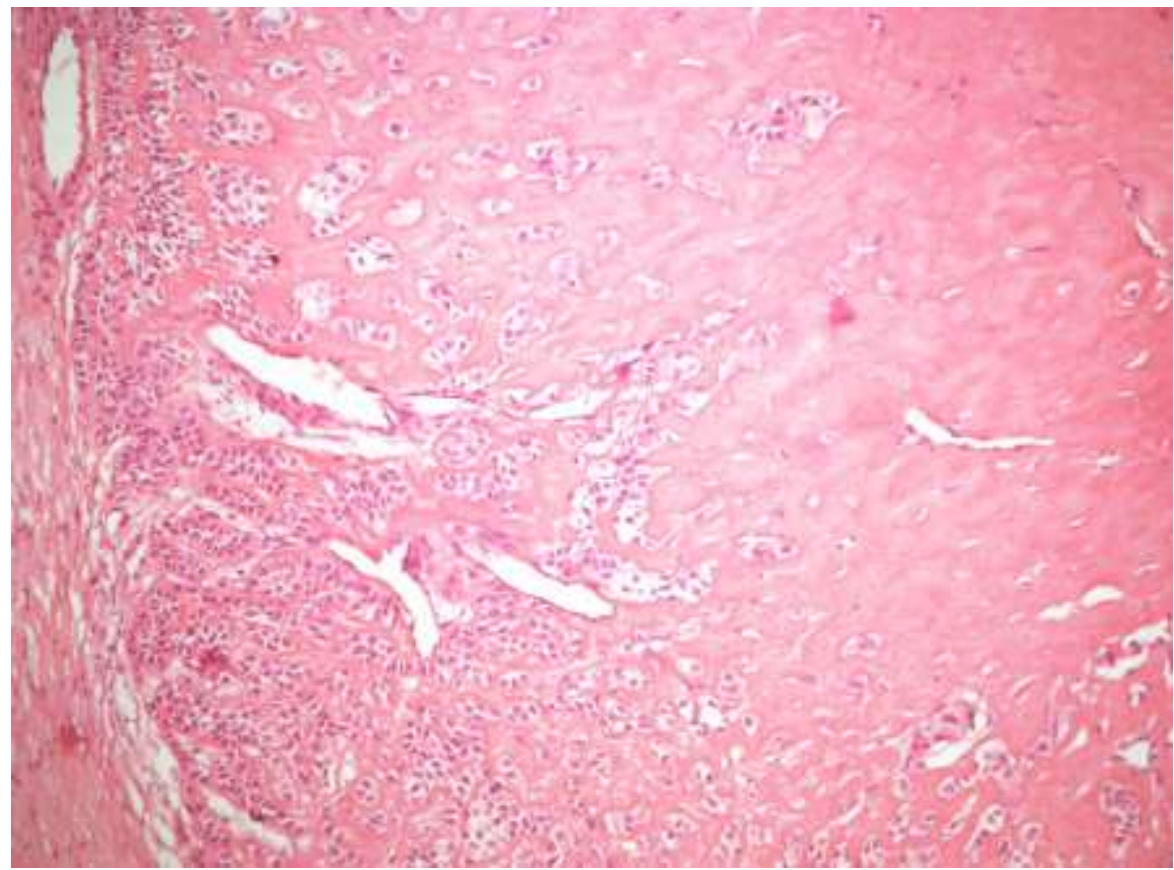

Figure 2:- photomicrograph (10x) showing hypercellular areas consisting of spindle shaped cells with duct like spaces. 


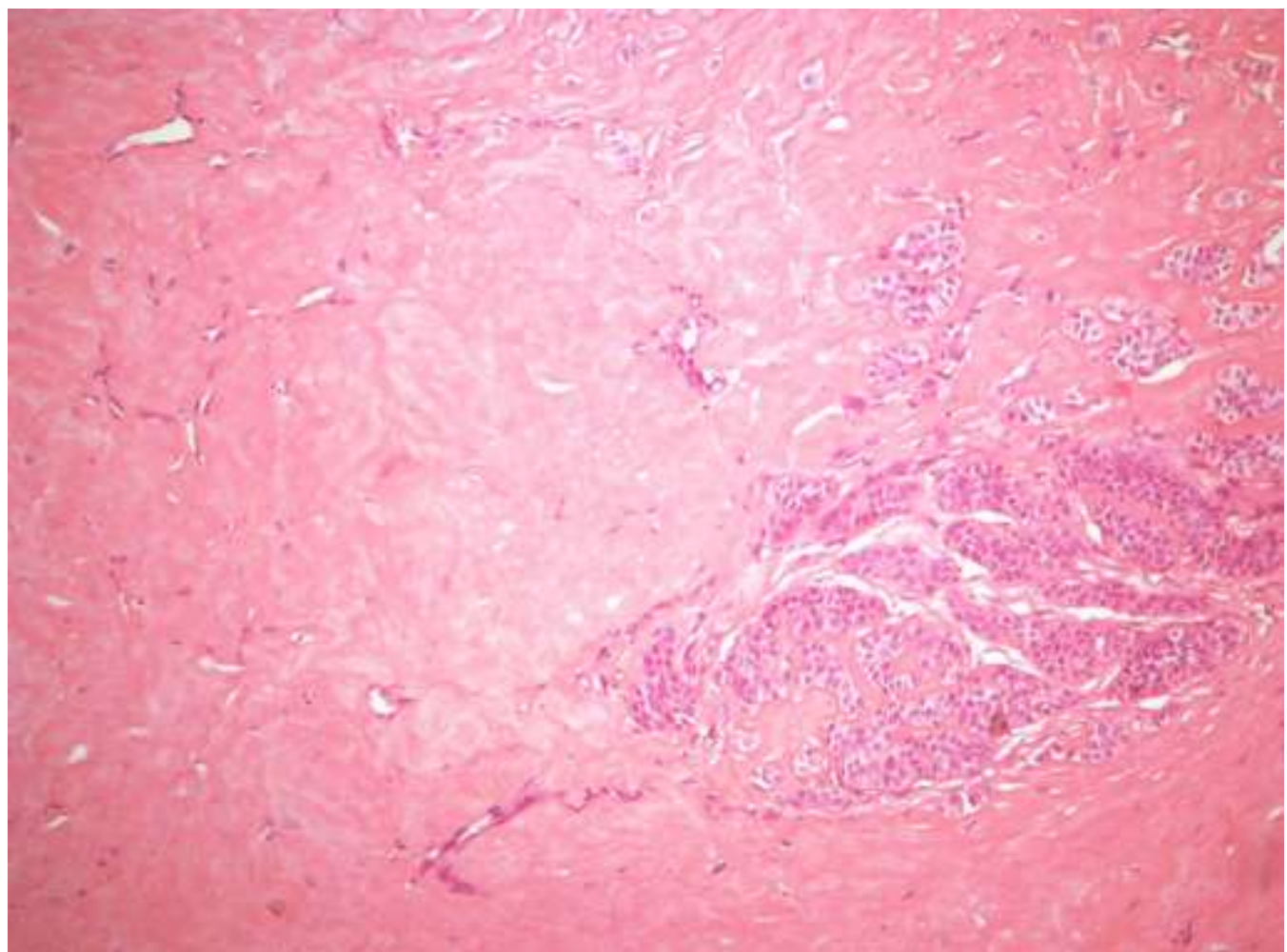

Figure 3:- Higher power view showing myxoid stroma along with spindle shaped myoepithelial cells arranged in the form of strands and groups.

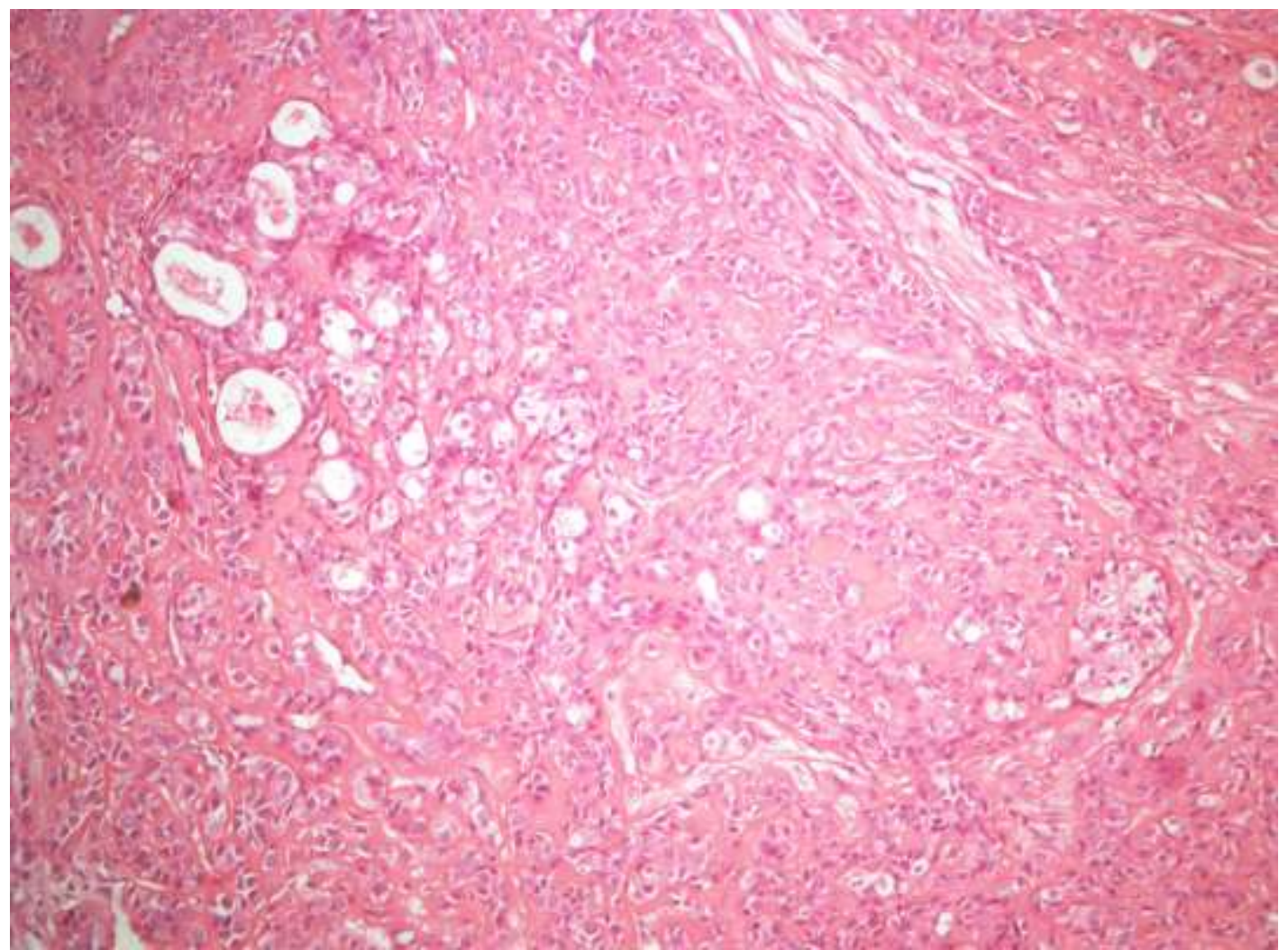

Figure 4:- Photomicrograph $(10 \mathrm{x})$ showing myxoid stroma along with chondroid areas. 


\section{References:-}

1. Amit Aggarwal, Ravinder Singh, Soheyl Sheikh. Pleomorphic adenoma of minor salivary gland: A case report. Revista Sul-Brasileira de Odontologia. 2012Jan-Mar; 9(1):97-101.

2. Ajay Kumar Pillai, Mrinal Satpathy, Saksham Nahar, Swapnil Moghe. Pleomorphic Adenoma in Cheek: An Uncommon Finding. IJSS Case Reports \& Reviews.2014;1(1):19-22..

3. Arpit Sharma, MS, DNB, Shraddha Deshmukh, MS, Ahmed Shaikh, MS, Jyoti Dabholkar MS. Pleomorphic adenoma of the minor salivary gland of the cheek, Singapore Med J 2013; 54(9): e183-e184.

4. Yen-Ling Kuo, et al. Extra - major salivary gland pleomorphic adenoma of the head and neck: A 10 - year experience and review of the literature. European archives of oto- rhino - laryngology. 2011;268(7): 10351040.

5. S Jain, S Hasan, N Vyas, N Shah, and S Dalal. Pleomorphic Adenoma of the Parotid Gland: Report of a Case With Review of Literature. Ethiop J Health Sci. 2015 Apr; 25(2): 189-194.

6. Mubeen K, Vijayalakshmi K. R, Abhishek Ranjan Patil, Girish B. Giraddi and Chandravir Singh. Benign pleomorphic adenoma of minor salivary gland of palate. Journal of Dentistry and Oral Hygiene 2011;3(6): 8288 .

7. Simon M.-Y. Lou, Alison M. Rich, Rohana K. De Silva, Martin M. Ferguson. Pleomorphic adenoma of a molar salivary gland. Oral oncology extra. 2006;42(4):170-172.

8. Taufik Dalati and Mahmoud R Hussein. Juvenile pleomorphic adenoma of the cheek: A case report and review of literature. Diagnostic Pathology2009;4(32):1-5.

9. Simon M.-Y. Lou, Alison M. Rich, Rohana K. De Silva, Martin M. Ferguson. Pleomorphic adenoma of a molar salivary gland. Oral Oncology EXTRA 2006;42:170- 172.

10. Pradhuman Verma, Suresh K Sachdeva, Kanika Gupta Verma, Kompal Sachdeva. Pleomorphic adenoma of cheek: A rare case report and review of literature. Indian Journal of Dental Research 2014; 25(1): 122-124.

11. Shubhangi Khandekar, Alka Dive, Prashant Munde, Neena Dongre Wankhede. Pleomorphic adenoma of the buccal salivary gland. Journal of Oral and Maxillofacial Pathology 2015;19(1).

12. Jain S, Hasan S, Vyas N, Shah N, Dalal S. Pleomorphic adenoma of the parotid gland: Report of a case with review of literature. Ethiop J Health Sci. 2015; 25(2): 189-194. 